\title{
PURELY INDECOMPOSABLE TORSION-FREE GROUPS
}

\section{PHILLIP GRIFFITH ${ }^{1}$}

1. Introduction. We consider the following question posed by E. Weinberg [8]: Does there exist a torsion-free abelian group of power greater than the continuum (N) with the property that each pure subgroup is indecomposable? J. W. Armstrong [1] has given a negative answer to this question for a class of groups somewhat larger than the class of homogeneous groups. In $\$ 2$ we show that in general the answer to Weinberg's question is negative. Furthermore we show that every reduced torsion-free purely indecomposable group is isomorphic to a subgroup of $J=\prod_{p} I_{p}$ where $p$ ranges over the primes and $I_{p}$ denotes the $p$-adic group. In $\S 3$ we characterize the pure subgroups of $J$ which are purely indecomposable and in $\$ 4$ we show there are exactly $2^{\aleph}$ nonisomorphic torsion-free purely indecomposable groups.

Throughout this note all groups are abelian and we follow [4] in notation. The notion of a cotorsion group introduced by Harrison in [7] plays an important role in this paper. Some basic properties of cotorsion groups are listed in [5].

Definition. A purely indecomposable group is a group in which every pure subgroup is indecomposable.

\section{The power of a torsion-free purely indecomposable group.}

LEMMA. Let $E$ be a torsion-free cotorsion group and let $x$ be an element of $E$. Then there is a direct summand of $E$ containing $x$ of power not exceeding $\mathbf{\aleph}$.

Proof. It suffices to consider $x \neq 0$. Let $A=\{x\}_{*}$, the pure closure in $E$ of the cyclic group $\{x\}$. Then $A$ is of rank one, which implies $|A|=\boldsymbol{\aleph}_{0}$. Now $E / A$ is torsion-free since $A$ is a pure subgroup of $E$. Therefore, $E / A=H / A+K / A$ where $K / A$ is reduced and torsionfree and $H / A$ is torsion-free divisible. It follows that

$$
E / H=E / A / H / A=K / A
$$

is a reduced torsion-free group. Since $E / H$ is reduced and $E$ is cotorsion, it is well known that $H$ must also be cotorsion. Thus $\operatorname{Ext}(E / H, H)=0$ and hence $H$ is a direct summand of $E$. Since $H / A$ is divisible, $A$ is

Received by the editors August 4, 1966.

1 The author wishes to acknowledge support by the National Aeronautics and Space Administration Grant NsG(T)-52. 
dense in the $n$-adic topology on $H$. However, the $n$-adic topology on a reduced torsion-free group is Hausdorff; thus it follows that $|H|$ $\leqq|A| \boldsymbol{\aleph}_{0}=\left(\boldsymbol{\aleph}_{0}\right) \boldsymbol{\aleph}_{0}=\mathbf{N}$.

THEOREM. If $G$ is a torsion-free purely indecomposable group, then the power of $G$ does not exceed $\aleph$.

Proof. If $G$ has a nontrivial divisible subgroup, it is immediate that $G$ must be isomorphic to the additive group of rational numbers if it is purely indecomposable.

Thus we may assume that $G$ is reduced and also that $G \neq 0$. Embed $G$ as a pure subgroup of a torsion-free cotorsion group $E$. Let $x \neq 0$ be an element of $G$. By the above lemma, there is a direct summand $H$ of $E$ such that $x \in H$ and $|H| \leqq \aleph$. Let $E=M+H$ and $S=M \cap G$ $+H \cap G$. Since $M, H$ and $G$ are pure subgroups of $E$ and since $E$ is torsion-free, it follows that $M \cap G$ is pure in $M$ and $H \cap G$ is pure in $H$. Thus, $E=M+H$ implies that $S$ is a pure subgroup of $E$; hence $S$ is a pure subgroup of $G$. Therefore, since $G$ is purely indecomposable, we have that $M \cap G=0$ or $H \cap G=0$. But $x \neq 0$ in $H \cap G$ implies that $M \cap G=0$. Let $\pi$ be the natural projection of $E=M+H$ onto $H$. Since $M \cap G=0, \pi$ restricted to $G$ is a monomorphism. Thus $|G|$ $=|\pi(G)| \leqq|H| \leqq \aleph$.

As in the introduction, let $J=\prod_{p} I_{p}$ where $p$ ranges over the primes and $I_{p}$ denotes the $p$-adic group. We have the following.

Corollary. If $G$ is a reduced, torsion-free, purely indecomposable group, then $G$ can be embedded in the $n$-adic completion of a reduced torsion-free group of rank one. Thus $G$ is isomorphic to a subgroup of $J$.

Proof. We use the notation in the proof of the above theorem. Since $H$ is a reduced torsion-free cotorsion group, it follows that $H$ is algebraically compact and thus complete in its $n$-adic topology. Since $H /\{x\}_{*}$ is divisible, $\{x\}_{*}$ is of rank one, and $\pi: G \rightarrow H$ is a monomorphism, the first part of the corollary is immediate. It is also known that $H=\prod_{p} A_{p}$ where, for each prime $p, A_{p}$ is a $p$-adic module complete in its $p$-adic topology. Each $p$-basic subgroup of $H$ is cyclic since $H /\{x\}_{*}$ is divisible and each $p$-basic subgroup of $\{x\}_{*}$ is cyclic. (For the notion of a $p$-basic subgroup, see [6].) Thus $A_{p}$ is indecomposable as a $p$-adic module. It follows that $A_{p}=0$ or $A_{p}=I_{p}$ and that $H$ is isomorphic to a direct summand of $J$, which completes the proof.

3. Pure subgroups of $J$ which are purely indecomposable. We now consider the natural question: when is a subgroup of $J$ purely indecomposable? 
We first remark that a reduced torsion-free group $G$ is isomorphic to a pure subgroup of $J$ if and only if every $p$-basic subgroup of $G$ is cyclic. The proof of this (well-known) result is accomplished by embedding $G$ in its cotorsion completion $E$ and an argument similar to the last half of the preceding corollary. Secondly, if $J$ is given the usual ring structure of componentwise addition and multiplication, then every endomorphism of $(J,+)$ is a left multiplication, that is, $\alpha \in$ End $J$ if and only if $\alpha$ is a mapping of the form $x \rightarrow \pi x$ where $\pi$ is a fixed element of $J$. Thus in case $G$ is isomorphic to a pure subgroup of $J$ we have the following characterization of pure indecomposability.

THEOREM. Suppose $G$ is a reduced torsion-free group such that each p-basic subgroup of $G$ is cyclic. Then $G$ is purely indecomposable if and only if, for each pure subgroup $S$ of $G$, the nonzero endomorphisms of $S$ are all monomorphisms.

Proor. The sufficiency is obvious, for if $G$ contains a pure subgroup $S$ which has a nontrivial direct decomposition then End $S$ contains a nontrivial projection. Thus suppose $G$ is a pure subgroup of $J$ and that $G$ is purely indecomposable. Let $S$ be a pure subgroup of $G$ and suppose $\alpha \neq 0 \in$ End $S$. Let $A$ denote the kernel of $\alpha$ (which is pure since $G$ is torsion-free) and $B$ the pure closure of the image of $\alpha$. Using vector notation for elements of $J$, let $\Lambda=$ [primes $p \mid$ some $a \in A$ has a nonzero $p$ th coordinate] and let $\rho$ be the projection of $J$ onto $\prod_{p \in \Lambda} I_{p}$. To show that $A \cap B=0$ and that $A+B$ is a pure subgroup of $G$, it suffices to show that $\rho$ is the identity when restricted to $A$ and the zero homomorphism when restricted to $B$. Since $I_{p}$ is an integral domain, $\rho$ is the identity on $A$ and zero on $B$ if $a \cdot b=0$ for all $a \in A$ and all $b \in B$. Let $a=\left\langle a_{p}\right\rangle \in A$ and $b=\left\langle b_{p}\right\rangle \in B$. Since $S$ is a pure subgroup of $J$ and since $J$ is cotorsion, $\alpha$ can be extended to $\bar{\alpha} \in$ End $J$. Then $\bar{\alpha}=L_{\pi}$ for some $\pi \in J$ where $L_{\pi}$ denotes left multiplication by $\pi=\left\langle\pi_{p}\right\rangle$. Therefore, $0=L_{\pi}(a)=\pi a=\left\langle\pi_{p} a_{p}\right\rangle$. Thus $a_{p}=0$ for each prime $p$ such that $\pi_{p} \neq 0$. If $x=\left\langle x_{p}\right\rangle \in \operatorname{Im} L_{\pi}$, then clearly $x_{p}=0$ for each prime $p$ such that $\pi_{p}=0$. It follows that $a \cdot x=0$ if $x \in \operatorname{Im} \alpha$, and that there is an integer $n$ such that $n(a \cdot b)=a(n b)=0$ since $B=(\operatorname{Im} \alpha)_{*}$. Thus $a b=0$ since $J$ is torsion-free, and it follows that $A \cap B=0$ and $A+B$ is a pure subgroup of $G$. Therefore, $\operatorname{Ker} \alpha=A=0$ since $B=(\operatorname{Im} \alpha)_{*} \neq 0$.

In view of a result of Baer [4, Lemma 46.3], the above theorem is easily seen to remain true if the condition on the $p$-basic subgroups of $G$ is replaced by the condition that $G$ be homogeneous. Also, Armstrong [2] obtains similar results if $G$ is $p$-reduced and $p$-purely indecomposable. However, the property that pure subgroups have no 
nonzero endomorphisms except monomorphisms does not, in general, characterize purely indecomposable torsion-free groups. To establish this, we consider a result of Beaumont and Pierce [3] which states there is a subgroup $H$ of rank two of the two-dimensional rational vector space $V$ such that the ring of quasi-endomorphisms $E(H)$ of $H$ consists of all $2 \times 2$ triangular matrices over $V$ with equal diagonal elements. Such a group $H$ must be indecomposable and hence purely indecomposable since $H$ is of rank two. Moreover, End $H$ contains an endomorphism which is not a monomorphism since $E(H)$ does.

4. The power of the collection $\beta$ of all nonisomorphic torsion-free purely indecomposable groups. Since the number of subgroups of $J$ is less than or equal to $2^{\aleph}$, the corollary in $\$ 2$ implies that the power of $\rho$ is less than or equal to $2^{\aleph}$. Therefore to establish that the power of $\rho$ is actually equal to $2^{\aleph}$, we need only show the existence of $2^{\aleph}$ nonisomorphic torsion-free purely indecomposable groups. We proceed by first proving the following lemma.

LemMa. Let $S$ be a pure subgroup of $J$ and let $I(S)$ be the collection of all subgroups of $J$ which are isomorphic to $S$. Then $I(S)$ has power less than or equal to $\mathbf{N}$.

Proof. Let $H \in I(S)$ and let $\phi$ be an isomorphism of $S$ onto $H$. Then $\phi \in \operatorname{Hom}(S, J)$. Since $S$ is a pure subgroup of $J$ and since $J$ is cotorsion, $\phi$ can be extended to $\bar{\phi} \in$ End $J$. It follows that $|I(S)|$ $\leqq \mid$ End $J \mid=\boldsymbol{N}$.

THEOREM. There are $2^{\mathrm{N}}$ nonisomorphic pure subgroups of $J$ which are purely indecomposable. Thus, the collection $\beta$ of all nonisomorphic torsion-free purely indecomposable groups has power $2^{\aleph}$.

Proof. Since the rank of $I_{p}$ is $\boldsymbol{\aleph}$, let $\left[a_{p \lambda}\right]_{\lambda \in \Lambda}$ be an independent subset of $I_{p}$ where $|\Lambda|=\aleph$. For each $\lambda \in \Lambda$, define $a_{\lambda}=\left\langle a_{p \lambda}\right\rangle \in J$ $=\prod_{p} I_{p}$. Now the set $\left[a_{\lambda}\right]_{\lambda \in \Lambda}$ is an independent subset of $J$ since for any finite subset of $\left[a_{\lambda}\right]_{\lambda \in \Lambda}$ the elements are componentwise independent. Therefore, for every nonvoid subset $M \subseteq \Lambda$ define $H_{M}$ $=\sum_{\lambda \in M}\left\{a_{\lambda}\right\}$ and $G_{M}=\left(H_{M}\right)_{*}$. Let $h \neq 0 \in H_{M}$ where $h=\left\langle h_{p}\right\rangle \in J$ $=\prod_{p} I_{p}$. Then $h=n_{1} a_{\lambda_{1}}+n_{2} a_{\lambda_{2}}+\cdots+n_{k} a_{\lambda_{k}}$ where $\lambda_{1}, \cdots, \lambda_{k} \in M$ and where $n_{i} \neq 0$ for $i=1,2, \cdots, k$. Therefore $h_{p}=n_{1} a_{p \lambda_{1}}+\cdots$ $+n_{k} a_{p \lambda_{k}} \neq 0$ for each $p$ since $\left[a_{p \lambda}\right]_{\lambda \in \Lambda}$ is an independent subset of $I_{p}$ and $n_{i} \neq 0$ for $i=1,2, \cdots, k$. Thus if $g \neq 0 \in G_{M}$ and $g=\left\langle g_{p}\right\rangle$, then $g_{p} \neq 0$ for each $p$ since $G_{M}=\left(H_{M}\right)_{*}$.

Let $S \neq 0$ be a pure subgroup of $G_{M}$ and $\alpha \in$ End $S$ such that $\alpha(x)=0$ for some $x \neq 0 \in S$. Since $S$ must be a pure subgroup of $J$ and 
$\alpha \in \operatorname{Hom}(S, J)$, then $\alpha$ can be extended to $L_{\pi} \in$ End $J$ where $\pi=\left\langle\pi_{p}\right\rangle$ $\in J$ and $L_{\pi}$ denotes left multiplication by $\pi$. Therefore, $0=L_{\pi}(x)$ $=\pi x=\left\langle\pi_{p} x_{p}\right\rangle$ implies $\pi_{p} x_{p}=0$ for each $p$. Hence $\pi_{p}=0$ for each $p$ since we have shown that a nonzero element of $G$ has the property that each of its components are nonzero. Thus $\pi=0$ and therefore $\alpha=0$. This establishes that every nonzero endomorphism of $S$ must be a monomorphism. Hence, by our theorem in $\S 3, G_{M}$ is purely indecomposable for every nonvoid subset $M$ of $\Lambda$.

Let $g$ denote the collection of groups $G_{M}$ where $M$ ranges over all nonvoid subsets of $\Lambda$. By the independence of $\left[a_{\lambda}\right]_{\lambda \in \Lambda}$ we have that $G_{M}=G_{M^{\prime}}$ (in the set-theoretic sense) if and only if $M=M^{\prime}$. Thus $\mathcal{G}$ is a collection of pure subgroups of $J$ which are purely indecomposable, and $|g|=2^{\aleph}$. By our preceding lemma, $\left|I\left(G_{M}\right)\right| \leqq \aleph$ for each $G_{M} \in \mathcal{G}$. Since $|\mathrm{g}|=2^{\aleph}$, it follows that there are $2^{\aleph}$ nonisomorphic pure subgroups of $J$ which are purely indecomposable.

A slight generalization of the construction used in the preceding theorem indicates that two torsion-free purely indecomposable groups may be quite different in nature. Indeed, if $N$ and $M$ are two nonvoid subsets of the primes with $N$ finite, then one may construct a torsionfree purely indecomposable group $G$ with the following two properties:

(1) for $p \in N$, each $p$-basic subgroup of $G$ has rank greater than one.

(2) For $p \in M, G_{p}^{1} \neq 0$ where $G_{p}^{1}=\bigcap_{n<\omega} p^{n} G$.

Finally we see that if $N$ is nonempty and $M$ is taken to be the set of all primes $p$, then such a group $G$ as described above is neither isomorphic to a pure subgroup of $J$ nor is it isomorphic to a subgroup of $I_{p}$ for any prime $p$.

\section{REFERENCES}

1. J. W. Armstrong, On the indecomposability of torsion-free Abelian groups, Proc. Amer. Math. Soc. 16 (1965), 323-325.

2. —_ "On $p$-pure subgroups of the p-adic integers," in Topics in Abelian groups, Scott, Foresman and Co., Chicago, Ill., 1963; pp. 315-321.

3. R. A. Beaumont and R. S. Pierce, Torsion-free groups of rank two, Mem. Amer. Math. Soc. No. 38 (1961), 41 pp.

4. L. Fuchs, Abelian groups, Hungarian Academy of Sciences, Budapest, 1958.

5. - "Recent results and problems in Abelian groups," in Topics in Abelian groups, Scott, Foresman and Co., Chicago, Ill., 1963; pp. 12-13.

6. - Note on Abelian groups. II, Acta. Math. Acad. Sci. Hungar. 11 (1960), 117-125.

7. D. K. Harrison, Infinite Abelian groups and homological methods, Ann. of Math. 69 (1959), 366-391. 199.

8. E. Weinberg, Free lattice-ordered ahelian groups, Math. Ann. 151 (1963), 187- 